\title{
Effectiveness and safety of focused ultrasound ablation surgery compared with radiofrequency ablation in primary hepatocellular carcinoma treatment: a meta-analysis
}

\author{
Jian-Ying Zhang ${ }^{1}$, Long Chen ${ }^{2}$, Rong $\mathrm{Ma}^{3}$, Chun-Mu Miao ${ }^{3}$, Yun-Bing Wang ${ }^{3}$ \\ ${ }^{1}$ Department of Radiology, Chongqing General Hospital, University of Chinese Academy of Sciences, Chongqing, China; ${ }^{2}$ Department of \\ Hepatobiliary Surgery, Pidu District People's Hospital, Chengdu, China; ${ }^{3}$ Department of Hepatobiliary Surgery, The Second Affiliated Hospital of \\ Chongqing Medical University, Chongqing, China \\ Contributions: (I) Conception and design: JY Zhang, L Chen, R Ma, YB Wang; (II) Administrative support: None; (III) Provision of study materials or \\ patients: None; (IV) Collection and assembly of data: JY Zhang, L Chen, CM Miao, YB Wang; (V) Data analysis and interpretation: All authors; (VI) \\ Manuscript writing: All authors; (VII) Final approval of manuscript: All authors. \\ Correspondence to: Yun-Bing Wang. Department of Hepatobiliary Surgery, The Second Affiliated Hospital of Chongqing Medical University, \\ Chongqing, China. No. 76, Linjiang Road, Yuzhong District, Chongqing 400010, China. Email: wangyunbing@cqmu.edu.cn.
}

Background: The effectiveness and safety of focused ultrasound ablation surgery (FUAS) for primary hepatocellular carcinoma (HCC) treatment has not been fully evaluated. This study analyzed the effectiveness and safety of FUAS compared to radiofrequency ablation (RFA).

Methods: Studies published before November 1, 2020, in the following databases were analyzed: PubMed, Embase, Cochrane Library, Web of Science, Wanfang Data, CqVip, China National Knowledge Infrastructure (CNKI), and Chinese Biomedical (CBM) database. All publications were reviewed independently by two authors. Both randomized controlled trials (RCTs) and cohort studies examining the effectiveness and safety of FUAS and RFA were considered. RCTs and cohort studies' methodological quality were evaluated using the Cochrane collaboration tool and the Newcastle-Ottawa Scale, respectively.

Results: A total of 6,597 records were identified, from which 3 cohort studies were selected for quantitative synthesis. All studies had relatively high methodological quality. The meta-analysis indicated that FUAS and RFA had comparable 3-month overall survival (OS) rates [risk ratio (RR): 0.99, 95\% confidence interval (CI): 0.86 to 1.14 ], 6-month OS rates (RR: 1.03 , 95\% CI: 0.82 to 1.29 ), and 1-year OS rates (RR: $0.96,95 \%$ CI: 0.84 to 1.11 ). Also, individual studies reported that the tumor response (reflected by tumor response and tumor ablation rate) and posttreatment complications were comparable between patients treated with FUAS and patients treated with RFA. Due to the limited number of studies reporting tumor response and posttreatment complications, further meta-analyses could not be conducted.

Discussion: FUAS and RFA were comparable in terms of effectiveness and safety in the treatment of primary HCC. However, current evidence is limited, and more prospective RCTs are warranted to confirm these findings.

Keywords: Focused ultrasound ablation surgery (FUAS); radiofrequency ablation (RFA); primary; hepatocellular carcinoma (HCC); meta-analysis

Submitted Dec 18, 2020. Accepted for publication Apr 02, 2021.

doi: $10.21037 /$ tcr-20-3458

View this article at: http://dx.doi.org/10.21037/tcr-20-3458 


\section{Introduction}

Currently, surgery is considered the most effective treatment for patients with hepatocellular carcinoma (HCC) (1). However, surgery can be traumatic, and some patients cannot tolerate major surgical procedures (2). Local ablation, such as radiofrequency ablation (RFA), is a promising alternative treatment for HCC patients $(3,4)$. Compared with traditional surgery, RFA is minimally invasive, simple, and repeatable. Some studies have shown that RFA is comparable to surgical resection in its treatment efficacy for small HCCs $(5,6)$. Therefore, since 2005 , RFA has been recommended by various guidelines to treat selected patients with HCC (7). For example, RFA may be a preferable alternative for HCC patients who have a cirrhotic background or inferior liver function and cannot tolerate surgery (8). However, RFA has certain shortcomings in clinical practice. For instance, RFA requires puncture of the liver tissue, which may lead to a series of puncture-related complications, such as peritoneal bleeding and needle seeding of tumor cells $(9,10)$. Therefore, improved methods for the treatment of HCC are still wanting.

Over the years, the evolution of cancer treatment has gone through a transformation from invasive to noninvasive therapies. Focused ultrasound ablation surgery (FUAS) is a novel non-invasive treatment that may be less traumatic for patients with HCC (11). Unlike RFA, FUAS does not require fine-needle puncture, thereby effectively preventing puncture-related complications. FUAS uses high-intensity focused ultrasound (HIFU) equipment on targeting internal tumors, followed by the destruction of the tumor via heat, mechanical, or cavitation methods (12). The whole process of FUAS can be monitored by ultrasound or magnetic resonance imaging (MRI), and treatments can be adjusted in real-time by changing the focus via imaging. The clinical application of FUAS has developed rapidly and has been shown to have a considerable effect on survival in patients with HCC (13). However, published studies evaluating the effect of FUAS in the treatment of HCC have mostly been case reports or case series $(12,14)$, with few controlled studies (15). One vital reason is that the time for application of FUAS in HCC is not so long. Although the effect of FUAS in HCC is promising, it still needs time for the technique of FUAS to become mature. For patients, because many patients did not recognize this technique comprehensively, they might not select this new method as a treatment. For clinical practitioners, they also need time to master this new technique and then recommend it to the patients. For research institutions, they might not purchase the equipment for HIFU ablation and so they are unable to use this equipment in clinical trials.

A comprehensive search of the literature was performed to identify controlled studies examining FUAS and RFA in patients with HCC. We found each of the controlled studies included a small sample size (no more than 100 cases in each group). So, it is needed to perform a meta-analysis. This study will help to clarify the clinical value of FUAS in the treatment of HCC and provide novel directions for future research. We present the following article in accordance with the Preferred Reporting Items for Systematic Reviews and Meta-Analyses (PRISMA) reporting checklist (16) (available at http://dx.doi.org/10.21037/tcr-20-3458).

\section{Methods}

\section{Search strategy}

This meta-analysis was prospectively registered in the international prospective register of systematic reviews database (PROSPERO: CRD42020188288). Eight online databases, including the PubMed, Embase, Cochrane Library, Web of Science, Wanfang Data, CqVip, China National Knowledge Infrastructure (CNKI), and Chinese Biomedical (CBM) databases, were searched for controlled studies examining FUAS and RFA as treatments for primary HCC. Studies published before November 1, 2020, were screened. The search terms included "HIFU", "high-intensity focused ultrasound", "focused ultrasound", "focused ultrasound ablation", "focused ultrasound surgery", "focused ultrasound ablation surgery", "FUAS", "FUA", "RFA", "radiofrequency", "ablation, radiofrequency", "HCC", "hepatocellular carcinoma", "hepatoma", "carcinomas, hepatocellular" and so on. The Web of Science database was searched in the Subject field but not in the Full text field because the latter was not available when we selected "all databases". However, in the other databases, the search was conducted in the Full text field. No other limitations were set during the literature search. Finally, the reference lists of the included articles, reviews, and guidelines were manually searched for additional studies.

\section{Inclusion and exclusion criteria}

The literature was selected according to the following inclusion criteria: (I) studies where the individuals were clinically or pathologically diagnosed with primary HCC; (II) studies in which the patients underwent FUAS treatment (FUAS group) or RFA treatment (RFA group), 
where both FUAS and RFA were conducted percutaneously; (III) studies in which reported outcomes included effectiveness- or safety-related outcomes, such as overall survival (OS) rates, tumor response, and post-treatment complications; and (IV) studies which were randomized controlled trials (RCTs) or cohort studies.

The exclusion criteria were as follows: (I) animal studies; (II) studies that did not include FUAS and RFA as treatments for primary HCC; (III) studies not related to the investigation of HCC; (IV) studies using duplicated data from other investigations; and (V) articles including reviews, conference abstracts, or subject indexes.

\section{Study selection, data extraction, and quality assessment}

Two reviewers (JYZ and YBW) independently excluded duplicates and screened the titles, abstracts, and full texts for relevance. Inconsistent results were resolved by discussion. The following data were collected from the included literature: first author, publication year, country, study design, intervention method, sample size, and outcomes. The RCTs' methodological quality was evaluated using the Cochrane collaboration tool based on six domains (17). A cohort study is a type of observational research that divides a specific population into different subgroups according to whether they are exposed to certain factors. The differences in the incidence of outcomes between groups are then compared. The Newcastle-Ottawa Scale (18) was used to evaluate the methodological quality of cohort studies regarding population selection, comparability, and exposure evaluation. The scale used is a semi-quantitative star system, and the full score is 9 stars.

\section{Statistical analysis}

The study-specific risk ratio (RR) for the categorical variables was utilized to calculate the pooled value using the DerSimonian-Laird method. Studies that reported outcomes were selected for quantitative synthesis. Stata software (version 16.0) was used for data analysis. Forest plots were used to display the results of the meta-analysis visually. Heterogeneity between different studies was evaluated using the $\mathrm{I}^{2}$ statistic, where $\mathrm{I}^{2} \leq 50 \%, 50 \%<\mathrm{I}^{2}$ $<75 \%$, and $\mathrm{I}^{2} \geq 75 \%$ represents low, moderate, and high heterogeneity, respectively. When high heterogeneity was detected, the outcome would be combined using a randomeffects model. Otherwise, the fixed-effects model would be selected. Sensitivity analyses were conducted to assess the robustness of the synthesized results by changing the used effects model or excluding one study at a time in each meta-analysis. Funnel plots would be used to evaluate the publication bias if the number of included studies is greater than 10. Otherwise, publication bias was assessed by Egger's test and Begg's test. Subgroup analyses were conducted based on the total sample size ( $>50$ or not) and the publication year ( $>2010$ or not). A P value $<0.05$ was considered statistically significant.

\section{Results}

\section{Literature selection, characteristics, and methodological quality of the included studies}

The literature selection process is summarized in Figure 1. A literature search of the 8 databases resulted in 6,597 citations. After duplications were excluded, 5,782 citations were retained. Screening the titles and abstracts for relevance resulted in the exclusion of 5,775 citations. Finally, seven citations (19-25) were retained, and the full text was evaluated for further selection. One citation (25) was a review, and one citation (24) did not include a comparison between FUAS and RFA. A further two studies $(22,23)$ were excluded as they included patients with recurrent HCC. The reference lists of the included literature were also screened, but no additional studies were found. Finally, three reports (19-21) that matched our study's inclusion criteria were included in the meta-analysis.

The included reports were all cohort studies published between 2006 and 2019, and all originated from China. From these 3 studies, a total of 111 patients underwent FUAS, and 123 patients underwent RFA. In two studies $(20,21)$, the general traits, including age and gender, were not significantly different between the FUAS and RFA groups. In the other study (19), age was reported to be comparable between the FUAS group and the RFA group. Two studies $(20,21)$ found that the tumor size, tumor number, and Child-Pugh grade were comparable between the FUAS and RFA groups. The detailed characteristics of the included studies are shown in Table 1. All three included studies had relatively high methodological quality, with all cohort studies (19-21) achieving eight stars on the Newcastle-Ottawa scale. However, other potential mixed factors' influence was not controlled, which may have affected the outcomes. 


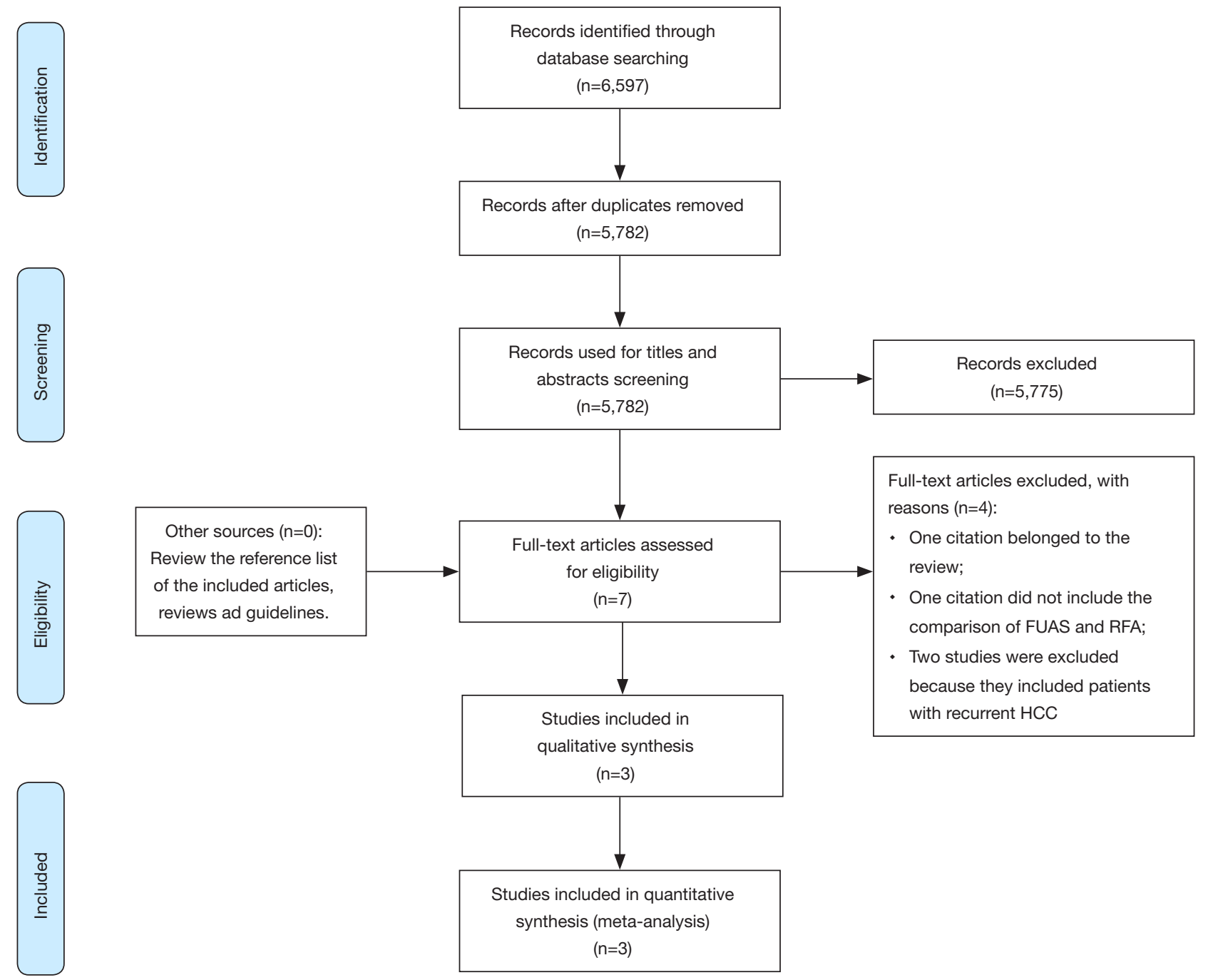

Figure 1 Flow chart of the literature selection process. Eight online databases were searched, and 6,597 citations were identified. After excluding duplicates, 5,782 citations were retained. Following a review of titles and abstracts, 5,775 citations were excluded as they did not match the inclusion criteria. A further 4 citations were excluded upon review of the full text articles. No other studies were found after reviewing the reference list of the included articles, reviews, and guidelines. Finally, 3 original studies were included in this meta-analysis.

\section{Primary outcomes}

\section{Meta-analysis of the 3-month OS rate}

A meta-analysis of two studies $(19,20)$ showed that the 3 -month OS rates in the FUAS group (87.04\%) and the RFA group $(87.93 \%)$ were not significantly different [RR $=0.99 ; 95 \%$ confidence interval $(\mathrm{CI}): 0.86$ to $1.14 ; \mathrm{P}=0.91$; Figure $2 A$ ], and there was no heterogeneity $\left(\mathrm{P}=0.62 ; \mathrm{I}^{2}=0\right)$.

\section{Meta-analysis of the 6-month OS rate}

A meta-analysis of two studies $(19,20)$ showed that the 6 -month OS rates in the FUAS group (72.22\%) and the
RFA group (70.69\%) were not significantly different (RR $=1.03 ; 95 \%$ CI: 0.82 to $1.29 ; \mathrm{P}=0.81$; Figure $2 B)$, and there was no heterogeneity $\left(\mathrm{P}=0.89 ; \mathrm{I}^{2}=0\right)$.

\section{Meta-analysis of the 1-year OS rate}

A meta-analysis of all three studies (19-21) showed that the 1 -year OS rates in the FUAS group (70.27\%) and the RFA group $(73.98 \%)$ were not significantly different $(\mathrm{RR}=0.96$; 95\% CI: 0.84 to $1.11 ; \mathrm{P}=0.59$; Figure $2 \mathrm{C}$ ), and there was no heterogeneity $\left(\mathrm{P}=0.84 ; \mathrm{I}^{2}=0\right)$. When subgroup analyses were conducted for studies with total sample size $>50$ or 


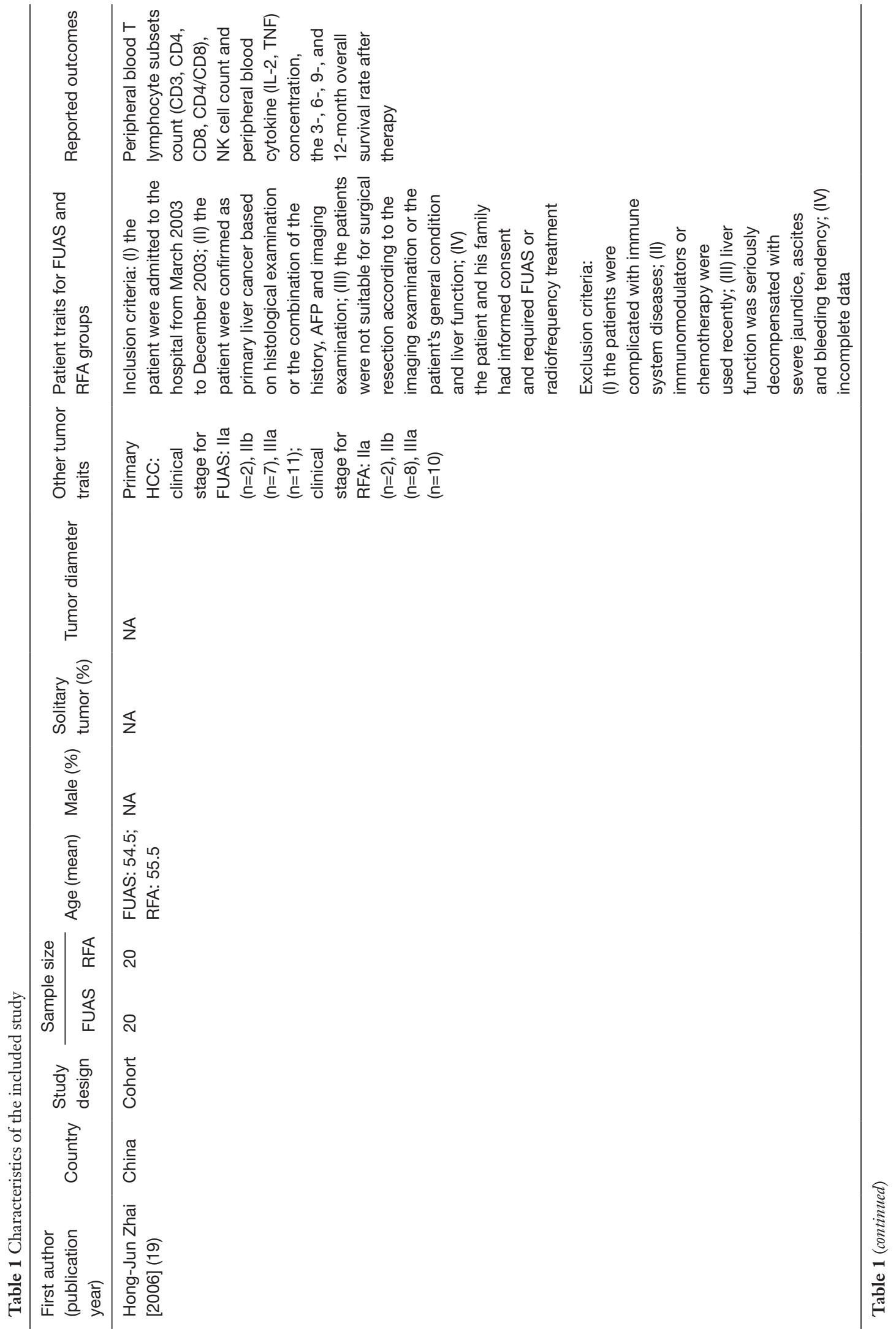




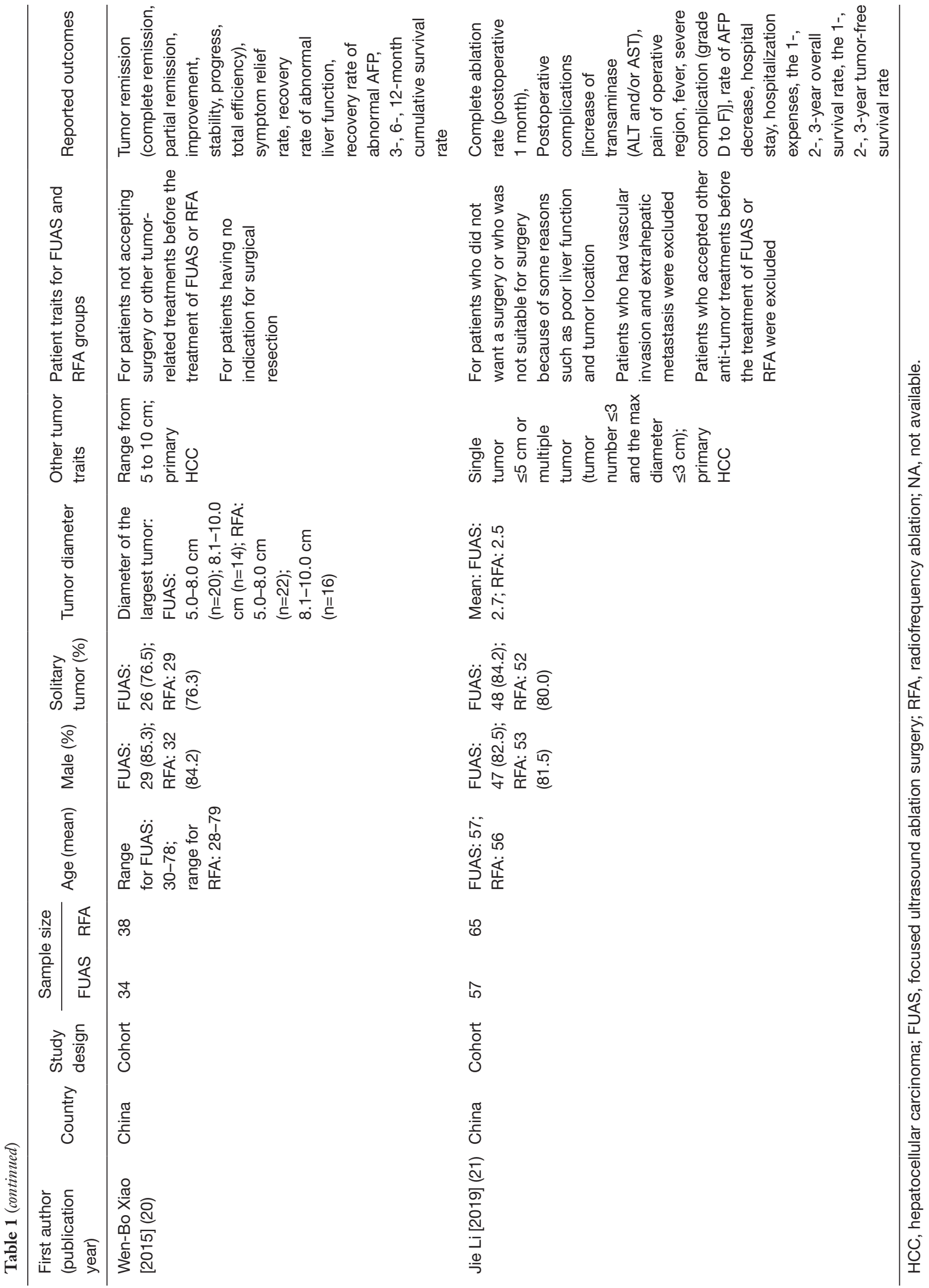


A

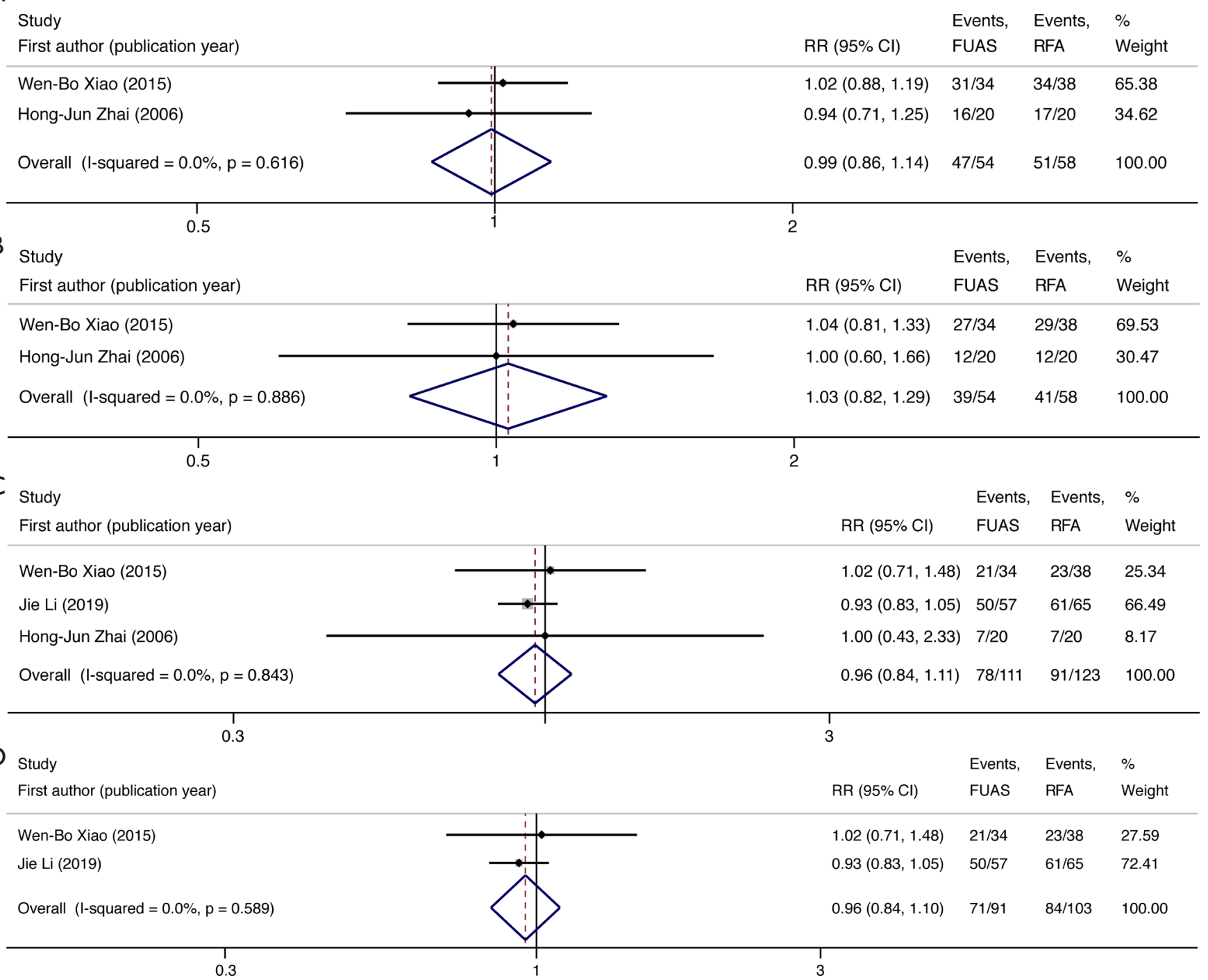

Figure 2 Meta-analysis of primary outcomes. Meta-analysis and subgroup analysis were conducted for primary outcomes. (A) The metaanalysis revealed that the 3-month OS in the FUAS group (87.04\%) and the RFA group (87.93\%) was not significantly different (RR $=0.99 ; 95 \%$ CI: 0.86 to $1.14 ; \mathrm{P}=0.91)$, with no heterogeneity $\left(\mathrm{P}=0.62 ; \mathrm{I}^{2}=0\right)$. (B) The meta-analysis revealed that the 6 -month $\mathrm{OS}$ in the FUAS group (72.22\%) and the RFA group (70.69\%) was not significantly different ( $\mathrm{RR}=1.03 ; 95 \%$ CI: 0.82 to $1.29 ; \mathrm{P}=0.81$ ), with no heterogeneity $\left(\mathrm{P}=0.89 ; \mathrm{I}^{2}=0 \%\right)$. (C) The meta-analysis revealed that the 1 -year OS in the FUAS group $(70.27 \%)$ and the RFA group $(73.98 \%)$ was not significantly different ( $R R=0.96 ; 95 \%$ CI: 0.84 to $1.11 ; \mathrm{P}=0.59)$, with no heterogeneity $\left(\mathrm{P}=0.84 ; \mathrm{I}^{2}=0 \%\right)$. (D) Subgroup analyses for studies with total sample size $>50$ or publication year $>2010$ indicated that FUAS and RFA showed no difference in the 1-year OS rate ( $\mathrm{RR}=0.96 ; 95 \%$ CI: 0.84 to $1.10 ; \mathrm{P}=0.53$; P for heterogeneity $=0.59 ; \mathrm{I}^{2}=0$ ). OS, overall survival; FUAS, focused ultrasound ablation surgery; $\mathrm{RFA}$, radiofrequency ablation; $\mathrm{RR}$, risk ratio; $\mathrm{CI}$, confidence interval.

publication year $>2010$, one study (19) was excluded and the other two studies $(20,21)$ were retained. The result of subgroup analyses indicated that FUAS and RFA showed no difference in the 1 -year OS rate ( $\mathrm{RR}=0.96 ; 95 \% \mathrm{CI}: 0.84$ to $1.10 ; \mathrm{P}=0.53 ; \mathrm{P}$ for heterogeneity $=0.59 ; \mathrm{I}^{2}=0$; Figure $2 D$ ).

\section{Secondary outcomes}

One study (21) found that the 1-year, 2-year, and 3-year tumor-free survival rates in the FUAS group were $81.2 \%$, $66.3 \%$, and $46.4 \%$, respectively. The corresponding survival 
rates in the RFA group were $84.5 \%, 72.1 \%$, and $52.1 \%$. After comparison, no differences in tumor-free survival rates were found between the FUAS and RFA groups. Using the WHO criteria, one study (20) compared the differences in tumor response, including complete response, partial response, stable disease, and progressive disease, and found no differences between the FUAS and RFA groups. Another study (21) analyzed the rate of complete ablation (1 month postoperatively) and found no difference between the FUAS group (90\%) and the RFA group (94\%). This latter study further analyzed and compared posttreatment complications, including increased transaminase levels, pain in the operative region, fever, and severe complications (grade D to F). The severe complications reported in the FUAS group and the RFA group were third degree skin burns and abdominal hemorrhage, respectively. After statistical analysis, no differences in posttreatment complications were detected between the FUAS and RFA groups.

\section{Sensitivity analyses}

Changing the fixed-effects model to the random-effects model did not affect the meta-analysis results of the 3-month, 6-month, and 1-year OS rates. For the 1-year OS rate, excluding the studies, one at a time did not alter the meta-analysis conclusions.

\section{Publication bias}

As the number of included studies was less than ten, publication bias was not evaluated by funnel plots. Instead, publication bias was assessed by Egger's test and Begg's test. Considering the 1-year OS rate outcome, no publication bias was detected, as indicated by Egger's test $(\mathrm{P}=0.38)$ and Begg's test $(\mathrm{P}=1.00)$.

\section{Discussion}

As the prognosis of patients with HCC is still poor, there is a demand to develop better treatment strategies (26). FUAS is a newly developed non-invasive therapy that is usually applied to treat gynecological diseases such as uterine fibroids and adenomyosis of the uterus (27). With recent advances in FUAS technology, the clinical application of FUAS for the treatment of carcinomas has gradually increased (28). Following an extensive literature search, this study identified and reviewed three articles comparing
FUAS and RFA in the treatment of primary HCC. The limited number of reports identified reflects the limited use of FUAS in the treatment of HCC. One reason for this is that the clinical application of FUAS is still in its infancy. Also, there are some clinical factors that may limit the use of FUAS in HCC. For example, respiratory activity might cause the liver to move, which would interfere with the ultrasound location for FUAS.

Furthermore, the liver might be partly covered by the ribs, which could lead to ultrasonic attenuation. Recent efforts have resolved some of these issues (15). For example, gas interference in the pleural cavity can be reduced by establishing artificial chest water. The influence of rib occlusion on the liver can be reduced by improving the treatment scheme. The effects of liver movement can be reduced by controlling respiration. Therefore, as FUAS technology continues to advance, the application of FUAS in HCC will gradually increase.

The most common outcome for evaluating the longterm effectiveness of carcinoma treatments is the OS rate. There was no difference in the 3-month, 6-month, or 1 -year OS rates in patients treated with FUAS and patients treated with RFA. These results suggested that FUAS is effective at improving the OS of patients suffering from HCC. However, only one included study (21) compared the 2 -year and 3-year survival rates, and the other two studies $(19,20)$ compared the 1-year survival rates at the longest. Survival rates should be monitored over a longer period (2 years or more) to comprehensively assess the effects of FUAS in the treatment of HCC patients. The one study (21) that monitored the 2-year and 3-year OS rates found no differences between the FUAS group and the RFA group. This latter study was also the only included report to assess the tumor-free survival rate. Again, no differences in the 1-year, 2-year, and 3-year tumor-free survival rates were found between the FUAS and RFA groups. Although the literature studies were limited in exploring the difference between FUAS and RFA in the long-term effectiveness as reflected by the OS and tumor-free survival rate, the current evidence indicated that there is no difference between FUAS and RFA treatment in patients with HCC.

Tumor response is an indicator that reflects the shortterm effectiveness of certain local treatments and can be evaluated by well-defined criteria. The criteria have been updated from the early WHO criteria to Response Evaluation Criteria in Solid Tumors (RECIST), RECIST 1.1, and finally the modified RECIST (29). Among the included literature, one study (20) evaluated the tumor 
response using the $\mathrm{WHO}$ criteria. This study compared the differences in complete response, partial response, stable disease, and progressive disease and found that the FUAS and RFA groups showed no difference in tumor response. Another study (21) did not use any of the above criteria but instead analyzed the complete ablation rate and found no differences between the FUAS group and the RFA group. As the number of studies comparing tumor response between FUAS treatment and RFA treatment is limited, a meta-analysis could not be performed. However, the current evidence suggested that FUAS and RFA have comparable short-term effectiveness in the treatment of HCC.

To analyze the safety of FUAS and RFA in the treatment of HCC patients, posttreatment complications were examined. Only one study (21) analyzed and compared posttreatment complications as an outcome and found no differences between the FUAS group and the RFA group. Similarly, because of the limited reports describing posttreatment complications, no meta-analysis for this indicator was performed. However, the available data suggested that there is no difference in posttreatment complications between patients treated with FUAS and patients treated with RFA. Currently, the Clavien-Dindo grading system is commonly used to grade complications from various treatments (30). Future studies examining the posttreatment complications of FUAS and RFA in HCC should use the Clavien-Dindo grading system to allow effective evaluation of patient safety. Overall, analysis of the long-term effectiveness, short-term effectiveness, and safety in the published literature suggested that FUAS and RFA are comparable in the treatment of primary HCC.

There were some shortcomings in this meta-analysis. First, the total number of patients in the FUAS and RFA groups was 111 and 123, respectively. The small number of patients included might affect the conclusions of this study. Second, the studies included in this meta-analysis were all observational cohort studies. Although the patients' comparability in the FUAS and RFA groups was assessed using general and clinical characteristics of patients, these factors varied between the three studies and thus, may influence the study's conclusions. Future RCTs should be conducted to minimize the interference of these factors. Also, the time and energy intensity of FUAS and RFA applied in each study were not described in detail, and this might affect the evaluation of the therapeutic effects of the two techniques. Additionally, if surgery could be regarded as a third control group in this meta-analysis, the conclusion about the application of FUAS in HCC would be more reliable. However, after checking the included studies, we found all of them did not include surgery group. So, including one extra surgery group in such a metaanalysis is impossible. Future controlled studies including third surgery group would be expected. Furthermore, while hazard ratios (HR) are important for the meta-analysis of survival data, this could not be included in the current study, as the HR and $95 \%$ CI were not reported in the original studies. One study (21) used the survival curve to compare FUAS and RFA patients' OS. Calculations based on the survival curve revealed an HR of 0.56 and a corresponding $95 \% \mathrm{CI}$ of 0.18 to 1.78 . However, the HR and corresponding $95 \% \mathrm{CI}$ of the other two studies could not be calculated due to insufficient data. Future metaanalysis studies could be improved by including the HR data. Finally, the outcomes related to effectiveness and safety included in this study were relatively limited. This might lead to an incomplete evaluation of these parameters. Therefore, in the future, to better evaluate the effectiveness and safety of FUAS and RFA in the treatment of HCC, it is necessary to increase the sample size, optimize the research design, supplement treatment parameters, and examine more outcomes.

\section{Conclusions}

Taken together, the results of this meta-analysis suggested that FUAS is comparable to RFA in terms of long-term effectiveness, short-term effectiveness, and safety in the treatment of primary HCC. FUAS treatment is expected to become a promising treatment for HCC. However, to date, the evidence in the literature is limited. Therefore, more high-quality investigations are warranted further to evaluate the role of FUAS in HCC patients.

\section{Acknowledgments}

Funding: The study was funded by Chongqing Science and health joint medical research project (No. 2021MSXM139).

\section{Footnote}

Reporting Checklist: The authors have completed the PRISMA reporting checklist. Available at http://dx.doi. org/10.21037/tcr-20-3458

Conflicts of Interest: All authors have completed the ICMJE uniform disclosure form (available at http://dx.doi. 
org/10.21037/tcr-20-3458). The authors have no conflicts of interest to declare.

Ethical Statement: The authors are accountable for all aspects of the work in ensuring that questions related to the accuracy or integrity of any part of the work are appropriately investigated and resolved.

Open Access Statement: This is an Open Access article distributed in accordance with the Creative Commons Attribution-NonCommercial-NoDerivs 4.0 International License (CC BY-NC-ND 4.0), which permits the noncommercial replication and distribution of the article with the strict proviso that no changes or edits are made and the original work is properly cited (including links to both the formal publication through the relevant DOI and the license). See: https://creativecommons.org/licenses/by-nc-nd/4.0/.

\section{References}

1. Wilson GC, Geller DA. Evolving Surgical Options for Hepatocellular Carcinoma. Surg Oncol Clin N Am 2019;28:645-61.

2. Hackl C, Schlitt HJ, Renner P, et al. Liver surgery in cirrhosis and portal hypertension. World J Gastroenterol 2016;22:2725-35.

3. Thandassery RB, Goenka U, Goenka MK. Role of local ablative therapy for hepatocellular carcinoma. J Clin Exp Hepatol 2014;4:S104-11.

4. Li D, Kang J, Madoff DC. Locally ablative therapies for primary and metastatic liver cancer. Expert Rev Anticancer Ther 2014;14:931-45.

5. Shiina S, Sato K, Tateishi R, et al. Percutaneous Ablation for Hepatocellular Carcinoma: Comparison of Various Ablation Techniques and Surgery. Can J Gastroenterol Hepatol 2018;2018:4756147.

6. Zhu ZX, Huang JW, Liao MH, et al. Treatment strategy for hepatocellular carcinoma in China: radiofrequency ablation versus liver resection. Jpn J Clin Oncol 2016;46:1075-80.

7. Bruix J, Sherman M, Practice Guidelines Committee, et al. Management of hepatocellular carcinoma. Hepatology 2005;42:1208-36.

8. Giorgio A, Merola MG, Montesarchio L, et al. Percutaneous radiofrequency ablation of hepatocellular carcinoma in cirrhosis: analysis of complications in a single centre over 20 years. Br J Radiol 2017;90:20160804.

9. Chang S, Kim SH, Lim HK, et al. Needle tract implantation after percutaneous interventional procedures in hepatocellular carcinomas: lessons learned from a 10year experience. Korean J Radiol 2008;9:268-74.

10. Kim TH, Choi HI, Kim BR, et al. No-Touch Radiofrequency Ablation of VX2 Hepatic Tumors In Vivo in Rabbits: A Proof of Concept Study. Korean J Radiol 2018;19:1099-109.

11. Wang $Z$. The theoretical basis of minimally-invasive and non-invasive medicine: Treatments--Minimize harm to patients. Ultrason Sonochem 2015;27:649-53.

12. Ng KK, Poon RT, Chan SC, et al. High-intensity focused ultrasound for hepatocellular carcinoma: a single-center experience. Ann Surg 2011;253:981-7.

13. She WH, Cheung TT, Jenkins CR, et al. Clinical applications of high-intensity focused ultrasound. Hong Kong Med J 2016;22:382-92.

14. Wu F, Wang ZB, Chen WZ, et al. Extracorporeal high intensity focused ultrasound ablation in the treatment of 1038 patients with solid carcinomas in China: an overview. Ultrason Sonochem 2004;11:149-54.

15. Diana M, Schiraldi L, Liu YY, et al. High intensity focused ultrasound (HIFU) applied to hepato-bilio-pancreatic and the digestive system-current state of the art and future perspectives. Hepatobiliary Surg Nutr 2016;5:329-44.

16. Moher D, Liberati A, Tetzlaff J, et al. Preferred reporting items for systematic reviews and meta-analyses: the PRISMA statement. PLoS Med 2009;6:e1000097.

17. Higgins JPT, Green S. editors. Cochrane Handbook for Systematic Reviews of Interventions Version 5.1.0 [updated March 2011]. The Cochrane Collaboration, 2011.

18. Stang A. Critical evaluation of the Newcastle-Ottawa scale for the assessment of the quality of nonrandomized studies in meta-analyses. Eur J Epidemiol 2010;25:603-5.

19. Zhai HJ, Jiang LS, Li N. Effect of High Intensity Focused Ultrasound on Immunity of Patients with Advanced Primary Liver Cancer. Chin J Bases Clin General Surg 2006;13:178-80.

20. Xiao WB, Liu H. Comparison of the recent therapeutic effect for large primary liver cancer between radiofrequency ablation and high intensity focused ultrasound. J Clin Ultrasound in Med 2015;17:252-4.

21. Li J, Guo WZ, Zhao YF, et al. Efficacy comparison of high-intensity focused ultrasound, argon-helium cryoablation and radiofrequency ablation in treatment of small liver cancer. Chin J Hepat Surg (Electronic Edition) 2019;8:133-8.

22. Cheung TT, Fan ST, Chu FS, et al. Survival analysis of high-intensity focused ultrasound ablation in patients with 
small hepatocellular carcinoma. HPB 2013;15:567-73.

23. Chan AC, Cheung TT, Fan ST, et al. Survival analysis of high-intensity focused ultrasound therapy versus radiofrequency ablation in the treatment of recurrent hepatocellular carcinoma. Ann Surg 2013;257:686-92.

24. Liu YX. Clinical effect of High Intensity Focused Ultrasound in patients with liver cancer. Med Equip 2020;33:106-7.

25. Nishikawa H, Osaki Y. Comparison of high-intensity focused ultrasound therapy and radiofrequency ablation for recurrent hepatocellular carcinoma. Hepatobiliary Surg Nutr 2013;2:168-70.

26. Eggert T, Greten TF. Current Standard and Future Perspectives in Non-Surgical Therapy for Hepatocellular Carcinoma. Digestion 2017;96:1-4.

27. Ji Y, Hu K, Zhang Y, et al. High-intensity focused

Cite this article as: Zhang JY, Chen L, Ma R, Miao CM, Wang YB. Effectiveness and safety of focused ultrasound ablation surgery compared with radiofrequency ablation in primary hepatocellular carcinoma treatment: a meta-analysis. Transl Cancer Res 2021;10(5):2080-2090. doi: 10.21037/tcr-203458 ultrasound (HIFU) treatment for uterine fibroids: a metaanalysis. Arch Gynecol Obstet 2017;296:1181-8.

28. Hsiao YH, Kuo SJ, Tsai HD, et al. Clinical Application of High-intensity Focused Ultrasound in Cancer Therapy. J Cancer 2016;7:225-31.

29. European Association for The Study Of The Liver; European Organisation For Research And Treatment Of Cancer. EASL-EORTC clinical practice guidelines: management of hepatocellular carcinoma. J Hepatol 2012;56:908-43.

30. Li SS, Udelsman BV, Parikh A, et al. Impact of Postoperative Complication and Completion of Multimodality Therapy on Survival in Patients Undergoing Gastrectomy for Advanced Gastric Cancer. J Am Coll Surg 2020;230:912-24. 